\title{
Postural Compensation for Vestibular Loss
}

\author{
Fay B. Horak, PhD, PT \\ Department of Neurology, Oregon Health and Science University, Portland, Oregon
}

\begin{abstract}
To what extent can remaining sensory information and/or sensory biofeedback compensate for loss of vestibular information in controlling postural equilibrium? The primary role of the vestibulospinal system is as a vertical reference for control of the trunk in space, with increasing importance as the surface becomes increasingly unstable. Our studies with patients with bilateral loss of vestibular function show that vision or light touch from a fingertip can substitute as a reference for earth vertical to decrease variability of trunk sway when standing on an unstable surface. However, some patients with bilateral loss compensate better than others and we find that those with more complete loss of bilateral vestibular function compensate better than those with measurable vestibulo-ocular reflexes. In contrast, patients with unilateral vestibular loss who reweight sensory dependence to rely on their remaining unilateral vestibular function show better functional performance than those who do not increase vestibular weighting on an unstable surface. Light touch of $<100$ grams or auditory biofeedback can be added as a vestibular vertical reference to stabilize trunk sway during stance. Postural ataxia during tandem gait in patients with unilateral vestibular loss is also significantly improved with vibrotactile biofeedback to the trunk, beyond improvements due to practice. Vestibular rehabilitation should focus on decreasing hypermetria, decreasing an over-dependence on surface somatosensory inputs, increasing use of any remaining vestibular function, substituting or adding alternative sensory feedback related to trunk sway, and practicing challenging balance tasks on unstable surfaces.
\end{abstract}

\section{Keywords}

posture; compensation; adaptation; vestibular loss; rehabilitation

\section{Compensation}

Immediately after sudden bilateral or unilateral loss of vestibular function, patients are immediately ataxic, with severe postural instability. Over weeks and months, postural stability improves through the process of vestibular compensation that may include a greater reliance on the remaining sensory information, although more challenging tasks on unstable surfaces reveals residual instability. The extent of vestibular compensation, both spontaneous and in response to rehabilitation varies widely among individuals. Some patients, but not others, show great improvements in postural stability when using vision, light touch, remaining vestibular function, or sensory biofeedback. This paper will review several mechanisms for postural compensation after loss of vestibular function, illustrating differences among subjects, and discuss how rehabilitation can facilitate each subject's compensation by augmenting sensory information for postural stability.

Address of Corresponding Author: Department of Neurology, Oregon Health and Science University, 505 NW $185^{\text {th }}$ Ave, Beaverton, Oregon 97006, Phone: 503-418-2600, Fax: 503-418-2501, horakf@ ohsu.edu. 


\section{Hypermetria}

Postural and gait ataxia after vestibular loss is due to increased amplitudes of both reactive and anticipatory postural responses (hypermetria). Hypermetric postural responses, as demonstrated by the large size of muscle and center of pressure responses to surface translations in cats after bilateral labyrinthectory (Fig. 1A), lead to overbalancing and instability as observed in the trajectory of the body center of mass (Figure 1B). ${ }^{1}$ Similar postural hypermetria is also seen in human subjects after ototoxic loss of vestibular function as illustrated by the large size of surface reactive torques in response to velocities of surface translations (Fig. $1 \mathrm{C}$ and D). ${ }^{2}$ The latency and scaling of postural responses is not changed by loss of vestibular function, although the responses are hypermetric, consistent with a somatosensory trigger of automatic postural responses ${ }^{3}$. Thus, poorly compensated vestibular patients show postural instability partially because their proprioceptively triggered postural responses are too large, not because they lack postural responses. Vestibular hypermetria may result either from reduced cerebellar inhibition of the spinal motor system, for the loss of vestibular inputs reduces the drive to the inhibitory Purkinje cells, or from reactive synaptogenesis of somatosensory inputs to the vestibular nucleus after loss of vestibular drive (Fig. 2). The process of compensation may involve learning how to appropriately calibrate the size postural responses using remaining or augmented sensory information.

\section{Sensory Substitution}

To what extent can remaining sensory information compensate for the role of vestibular information in controlling postural equilibrium? Our studies have shown that some bilateral vestibular loss subjects are better than others in using their vision ${ }^{4}$, light touch on a stable surface $^{5}$ or their remaining vestibular function ${ }^{6}$ to substitute for missing vestibular information as a vertical reference for trunk orientation in space. For example, although all subjects with severe, bilateral loss of vestibular function are unable to stand on an fast, oscillating surface with eyes closed, half of the subjects we tested were immediately able to maintain their stability when their eyes were open (Fig. 3$)^{4}$. In fact, stability of the head and trunk orientation in space during surface translations was within normal limits in wellcompensated subjects but very large in poorly compensated subjects. Surprisingly, the wellcompensated vestibular patients had somewhat lower vestibulo-ocular reflex gains than poorly-compensated subjects, suggesting that small amounts of remaining, perhaps distorted, vestibular function is not an advantage for compensation.

Light touch appears to provide a similar sensory substitution reference to earth vertical as vision for subjects with bilateral vestibular loss. ${ }^{5}$ Less than 100 grams of light touch of a single fingertip, that provides a sensory reference for earth vertical, but not mechanical support, results in a similar amount of trunk and head stability as vision when standing on a rotating surface. ${ }^{5}$ In fact, light touch, like vision, immediately stabilizes posture primarily at the higher frequencies of postural sway in patients with vestibular loss, whereas they stabilize the lower frequencies in control subjects (Fig. 4). We found that the bestcompensated patients with unilateral loss of vestibular function were those who used their remaining vestibular function in the intact ear, rather than rely upon their vision or somatosensory function. ${ }^{6}$ When UVL subjects stood with eyes closed on a pseudo-randomly rotating surface, on average they depended upon vestibular function for postural orientation about $50 \%$ as much as age-matched control subjects. However, the ability to use vestibular information varied widely among UVL subjects, with some subjects able to depend upon their remaining vestibular function as much as controls with bilateral function while others had very little use of their remaining vestibular function for postural orientation. Subjects who were able to depend on their unilateral vestibular function rated their ADL 
performance $^{7}$ and balance confidence ${ }^{8}$ better than those who could not depend on vestibular information when eyes were closed on an unstable surface.

\section{Sensory Addition}

Augmenting sensory information for balance control by providing visual, auditory, electrotactile or vibrotactile BF of body sway has been shown to immediately reduce postural sway during stance and gait. ${ }^{9}$ However, the extent to which BF successfully improves balance depends on the individual subject. For example, we have shown that the largest reductions of postural sway from audio-BF occurred in subjects with the least vestibular function and that vestibular loss subjects benefit more than healthy controls when standing on a foam surface with eyes closed but not when standing on a firm surface. ${ }^{9}$ Also, both healthy and vestibular-loss subjects who tended to be visually-dependent improved the most from BF when their eyes were closed whereas those who tended to be somatosensorydependent improved the most from BF when they were standing on compliant foam. ${ }^{9}$ Thus, sensory BF appears to be used most in the conditions in which it is most needed.

The extent that BF is used may also depend upon how it is presented. Whereas audioBF is most successful when presented as a sigmoidal function of trunk sway, visual biofeedback of trunk sway is most successful when presented as a linear function of trunk sway. ${ }^{10}$ Simple alarms at threshold positions of body sway may be just as useful as more complex transformations of postural stability and require further study.

Although in some ways augmented sensory feedback using BF devices appears to substitute for missing vestibular information to improve postural stability, it is not clear whether the nervous system is really increasing sensory weighting or reliance on BF like it does the natural senses or $\mathrm{BF}$ is just adding sensory information to reduce sensory noise. We have found that audioBF primarily reduces postural sway at lower frequencies than practice or light touch, perhaps because long loop voluntary control are used for BF. A recent study uses a model of sensory weighting to show that even a very sophisticated vibrotactile BF devise reduces postural sway on an unstable surface primarily by reducing sensory noise and not by increasing sensory reweighting to a vestibular substitute. ${ }^{11}$

We recently showed that $\mathrm{BF}$ also can improve dynamic postural stability during gait in patients with unilateral vestibular loss. ${ }^{12}$ AudioBF of lateral trunk sway immediately and significantly reduces lateral body CoM and trunk displacements and stance width, although it does not improve the rate of motor learning. In fact, repeated practice of a difficult task, such as walking with narrow base of support and eyes closed results in significant improvement across a single session with retention of performance across two-weeks. However, despite the improved postural stability while using BF, this improvement was not retained without $\mathrm{BF}$. This is what would be expected if $\mathrm{BF}$ acted like natural sensory information; improvements made during practice with eyes open would not be expected to transfer to improved balance with eyes closed.

\section{Implications for Vestibular Rehabilitation}

Studies of postural control following vestibular loss have important implications for rehabilitation of balance disorders. To identify the effects of loss of vestibular function on postural stability, clinicians need to examine patients on unstable surfaces, particularly at medium velocities of surface tilts. Rehabilitation should focus on stabilizing the head and trunk in space since postural responses in the legs may be normal. Rehabilitation should also focus on reducing hypermetric postural responses that destabilize vestibular patients, rather than attempting to facilitate reduced, late or absent postural responses. 
The most powerful method for compensating for vestibular loss is sensory substitution or sensory addition. Although patients naturally compensate with remaining vestibular function, vision and touch onto stable surfaces, some patients compensate better than others. Every effort should be made to teach patients to use any remaining, useful vestibular function by practicing balancing on unstable surfaces without use of vision, since patients who can rely on their remaining vestibular function report the best functional recovery. A cane that provides the nervous system with an external reference to earth via light touch remains the most powerful sensory substitution tool, even more powerful than vision for stabilizing posture. ${ }^{5}$

Sensory biofeedback devices may be useful since the nervous system appears to take advantage of any additional information well correlated with postural sway, whether auditory, visual, or somatosensory, although the characteristics of the feedback signal needs to be optimized for each sense (i.e. linear versus sigmoidal coding) and for each subject (i.e. by normalizing for each subject's limits of stability and sensory thresholds). To date, it appears that biofeedback does not act like as vestibular substitution but rather as a "balance prosthesis'. That is, biofeedback adds relevant sensory information about body sway to increase the signal to noise ratio. Studies are needed to determine the extent to which longterm use of BF can result in faster, more automatic use of the additional sensory information. Like a balance prosthesis, biofeedback is useful while it is being applied and not afterwards, so efforts must be made to develop biofeedback systems that are unobtrusive and can be easily suspended when adequate surface or visual references, that provide the first line of sensory substitution, are available. In conclusion, rehabilitation for loss of vestibular function must be customized for each patient since studies are showing that individuals vary in their sensory dependence and how they compensate for vestibular loss. Control of postural orientation and equilibrium can be significantly improved in patients with bilateral or unilateral vestibular loss as long as it is considered a complex, sensorimotor skill that must be learned with appropriate feedback and active, context-specific training.

\section{Acknowledgments}

Supported by NIH grant from NIDCD 004082.

\section{References}

1. Inglis JT, Macpherson JM. Bilateral labyrinthectomy in the cat: effects on the postural response to translation. Journal of Neurophysiology Mar. 1995; 73(3):1181-91.

2. Horak, FB. Adaptation of automatic postural responses. In: Bloedel, J.; Ebner, TJ.; Wise, SP., editors. Acquisition of Motor Behavior in Vertebrates. Cambridge: MIT Press; 1996. p. 57-85.

3. Horak FB, Nashner LM, Diener HC. Postural strategies associated with somatosensory and vestibular loss. Exp Brain Res. 1990; 82:167-177. [PubMed: 2257901]

4. Buchanan JJ, Horak FB. Vestibular loss disrupts control of head and trunk on a sinusoidally moving platform. J Vestibular Res. 2001-2002; 11(6):371-89.

5. Creath R, Kiemel T, Horak F, Jeka JJ. Limited control strategies with the loss of vestibular function. Exp Brain Res. 2002; 145(3):323-33. [PubMed: 12136382]

6. Statler, K.; Wrisley, D.; Peterka, R.; Horak, F. Functional deficits are correlated with decreased reliance on vestibular information in people with chronic unilateral vestibular loss. [abstract]. Association for Research in Otolaryngology; Daytona Florida. Feb 22-26, 2004;

7. Cohen HS, Kimball KT. Development of the vestibular disorders activities of daily living scale. Arch Otolaryngol Head Neck Surg. 2000; 126:881-887. [PubMed: 10889001]

8. Powell LE, Myers AM. The Activities-specific Balance Confidence (ABC) Scale. J Gerontology A Biol Sci Med Sci. 1995; 50A:M28-34. 
9. Dozza M, Horak FB, Chiari L. Auditory biofeedback substitutes for loss of sensory information in maintaining stance. Exp Brain Res Mar. 2007; 178(1):37-48.

10. Dozza M, Chiari L, Hlavacka F, Cappello A, Horak FB. Effects of Linear versus Sigmoid Coding of Visual or Audio Biofeedback for the Control of Upright Stance. IEEE Transactions. 2006; 14(4):505-512.

11. Goodworth A, Wall C III, Peterka RJ. Influence of feedback parameters on performance of a vibrotactile balance prosthesis. IEEE Transactions. Submitted.

12. Dozza M, Wall C III, Peterka RJ, Chiari L, Horak FB. Effects of practicing tandem gait with and without vibrotactile biofeedback in subjects with unilateral vestibular loss. J Vestibular Res. 2007; 17(4):195-204. 
A.
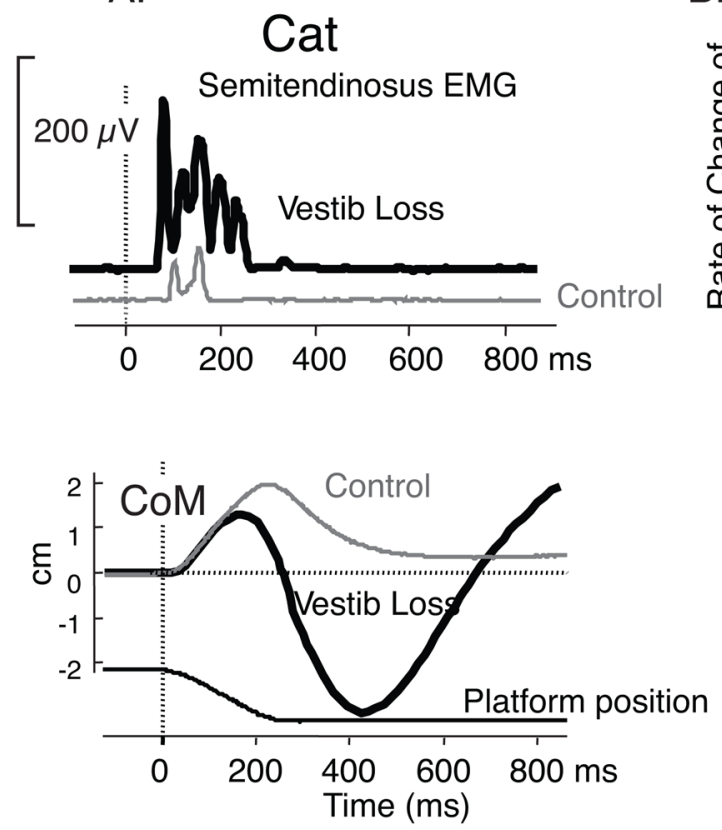

B.
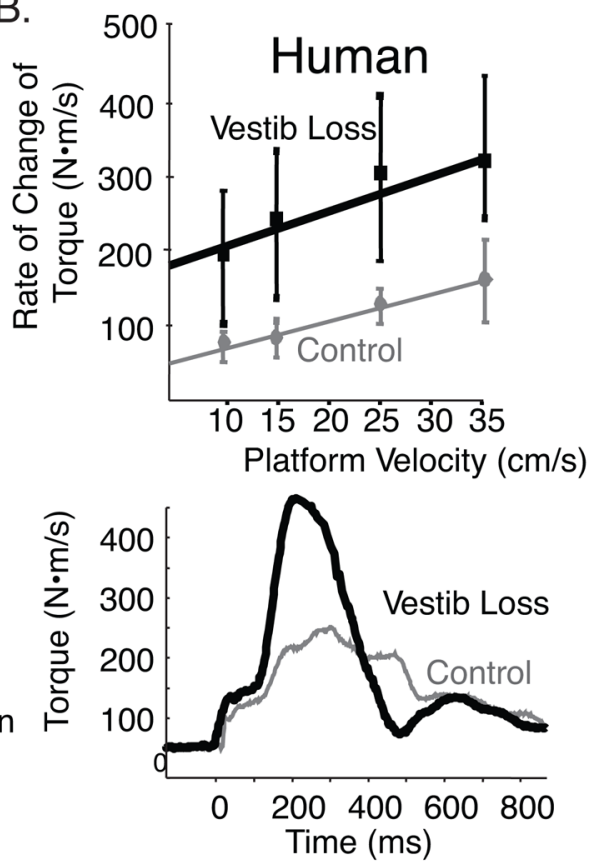

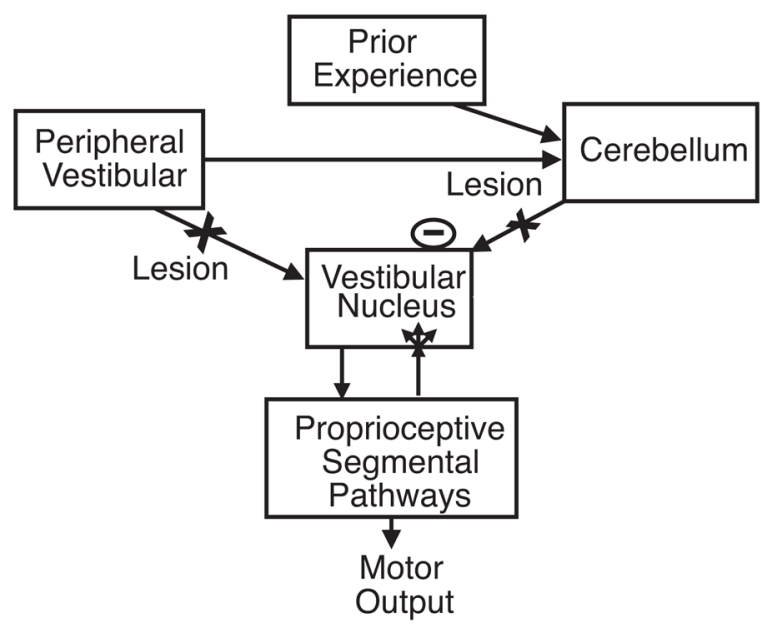

Figure 1.

Hypermetria of postural responses to surface translations in cats and humans with bilateral vestibular loss is illustrated. A. Example of semitendinosis EMG response and CoM and CoP response from 3 trials of forward-right diagonal translations in a cat before and after bilateral labyrinthectomy (adapted from Inglis and Macpherson, 1995). B. Group average of scaling surface reactive responses to increasing backward translation velocities from 7 subjects with chronic, bilateral vestibular loss due to ototoxicity and 7 age-matched control subjects and examples of surface reactive torques from a subject with bilateral labyrinthectomy compared with an age-matched control subject. Schematic of potential explanations for postural hypermetria after bilateral vestibular loss. Hypermetric proprioceptive-triggered postural responses could result either from synaptogenesis or increased efficacy of somatosensory inputs to the vestibular nucleus after loss of vestibular inputs or from loss of vestibular input to the cerebellum, resulting in loss of inhibitory drive to proprioceptive pathways involved in automatic postural responses. 


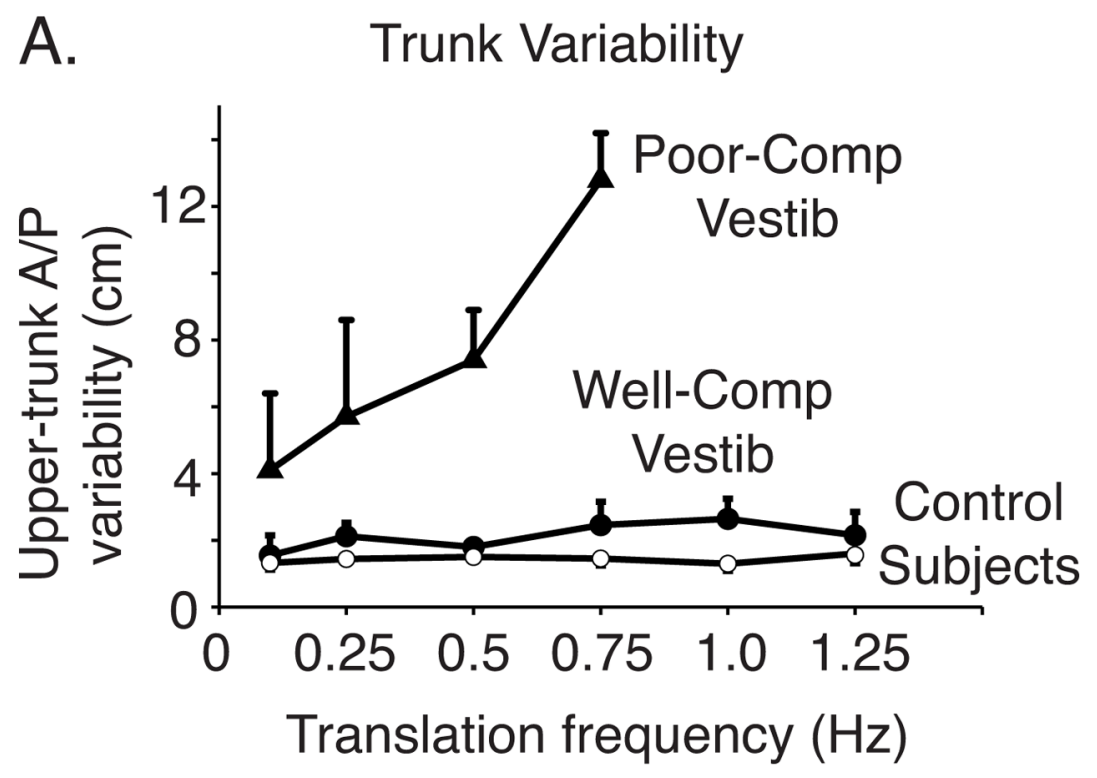

B.

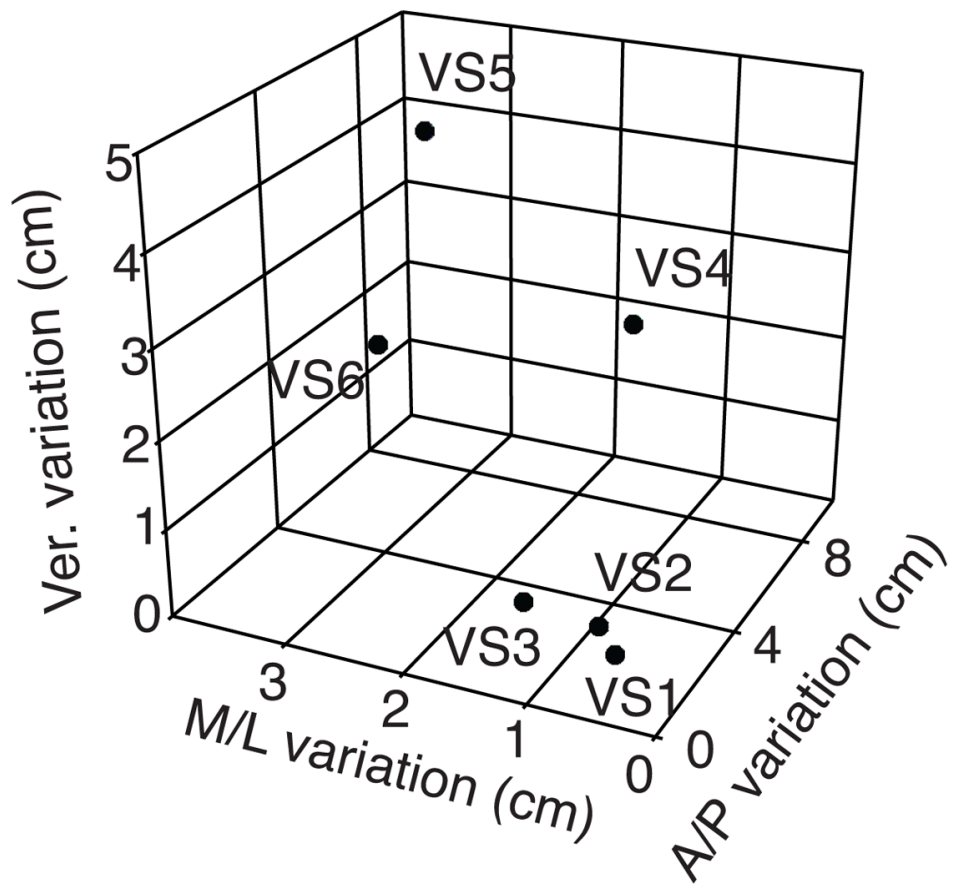

Figure 2.

A. Comparison of upper trunk orientation variability during sinusoidal surface translations with eyes open in 3 well-compensated, 3-poorly compensated vestibular loss subjects, and 10 control subjects. B. Comparison between 3 well-compensated and 3-poorly compensated vestibular loss subjects in head, trunk and leg orientation variation during sinusoidal surface translations with eyes open. Control subjects all showed head, trunk and leg orientation variation below $1 \mathrm{~cm}$. (Adapted from Buchanan and Horak, 2000). 


\section{CoM/Surface Displacement}

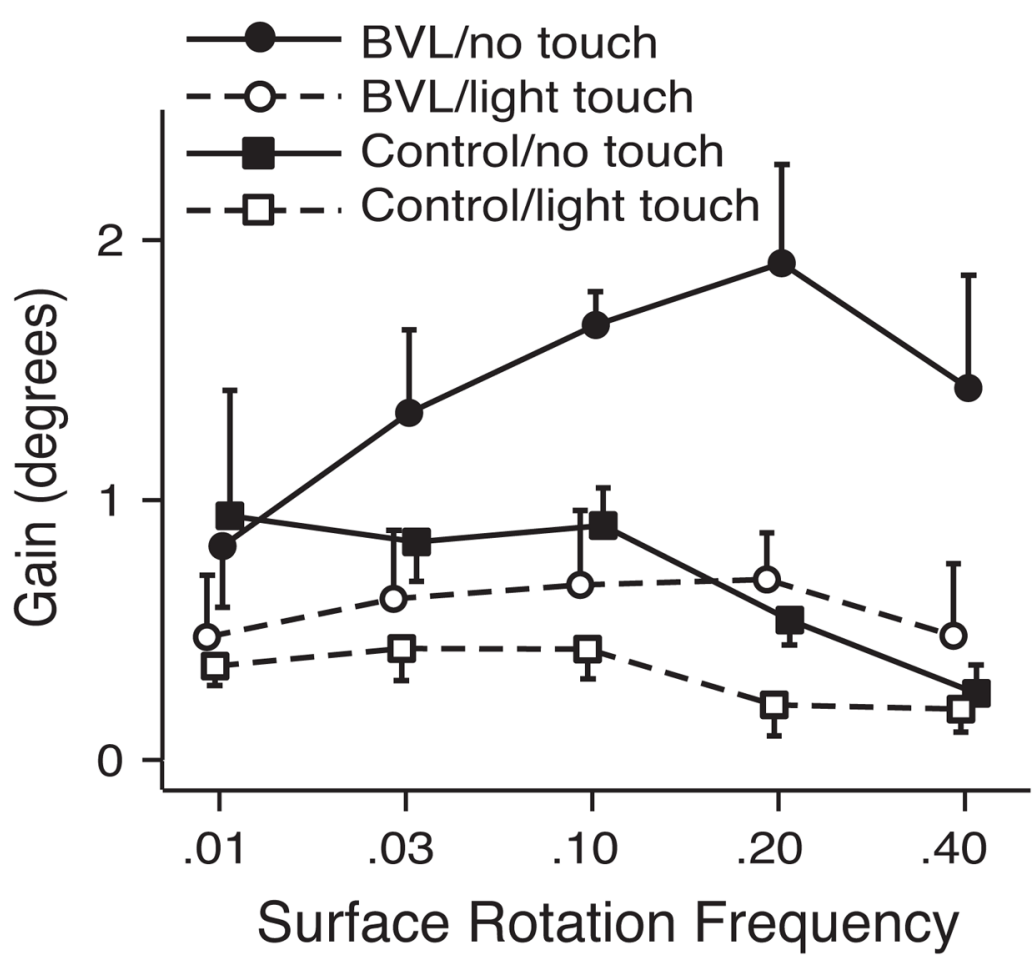

Figure 3.

Effects of light (<100 grams) finger tip touch on postural stability (gain-CoM displacement/ surface displacement) during.01, .03, .10, .20, .40 Hz sinusoidal surface rotation in seven subjects with bilateral vestibular loss and seven age-matched control subjects (adapted with permission from Creath, et al, 2002). Fingertip touch reduces postural sway more in subjects with vestibular loss than controls and subjects with vestibular loss benefit most at high frequencies of surface rotation whereas controls benefit most at low frequencies. 